\title{
Evaluating the association between walking speed and reduced cardio-cerebrovascular events in hemodialysis patients: a 7-year cohort study
}

\author{
Yoshifumi Abe ${ }^{1}$, Atsuhiko Matsunaga ${ }^{1 *}$, Ryota Matsuzawa ${ }^{2}$, Kei Yoneki ${ }^{1,3}$, Manae Harada ${ }^{1}$, Takaaki Watanabe $^{1}$, \\ Toshiki Kutsuna ${ }^{4}$, Masahiko Kimura ${ }^{5}$, Kyo Shigeta', Yasuo Takeuchi ${ }^{6}$ and Atsushi Yoshida ${ }^{3}$
}

\begin{abstract}
Background: Deterioration in walking ability characterized by slow walking speed is associated with an increased risk of mortality in patients on hemodialysis (HD), but few studies have examined the potential for walking speed to reduce the incidence of clinical events. In this study, we assessed a range of maximum walking speeds (MWS) with regard to their capacity to reduce cardio-cerebrovascular events in HD patients.

Methods: In total, 188 Japanese outpatients (90 men, 98 women; mean age, 65 years) undergoing maintenance HD three times a week were monitored for 7 years. We measured clinical characteristics and MWS at baseline and followed these patients to track any cardio-cerebrovascular events. Patients were divided into quartiles $(\mathrm{Q} 1=$ lowest, Q4 = highest) based on MWS for each sex. Cox proportional hazards regression and Kaplan-Meier estimates were used to assess the contribution of MWS to cardio-cerebrovascular events.

Results: During the follow-up period, cardio-cerebrovascular events occurred in 42 patients. After adjusting for potential confounders, the hazard ratio for events per $10 \mathrm{~m} / \mathrm{min}$ increase in MWS was 0.77 (95\% confidence interval: 0.65-0.92; $P=0.004$ ). Seven-year cumulative incidence rates were $36,32,13$, and $9 \%$ for Q1 through Q4, respectively, and a significant difference across MWS quartiles was observed $(P<0.001)$. While the incidence rates did not significantly differ between Q1 and Q2 or between Q3 and Q4, Kaplan-Meier curves clearly differed between Q2 and Q3.
\end{abstract}

Conclusions: Our findings suggest that more than the MWS level of Q3 ( $\geq 89 \mathrm{~m} / \mathrm{min}$ in men and $\geq 85 \mathrm{~m} / \mathrm{min}$ in women) may serve as important MWS values for disease management in ambulatory HD patients.

Keywords: Hemodialysis patient, Walking speed, Walking ability, Cardio-cerebrovascular event, Disease management

\section{Background}

Many recent clinical reports have demonstrated that motor function including walking ability can deteriorate significantly in patients with end-stage renal dysfunction who require hemodialysis (HD), compared to agematched community-based healthy individuals $[1,2]$. In

\footnotetext{
* Correspondence: atsuhikonet@gmail.com

'Department of Rehabilitation Sciences, Kitasato University Graduate School of Medical Sciences, 1-15-1 Kitasato, Sagamihara, Kanagawa 252-0373, Japan Full list of author information is available at the end of the article
}

particular, deterioration in walking ability characterized by slow walking speed is significantly associated with reduced physical activity, decline in instrumental activities of daily living, and overall lower quality of life [3-5]. Moreover, epidemiological cohort studies have found that slow walking speed is independently associated with mortality, hospitalization, and adverse events in healthy older individuals and in patients with heart disease, kidney disease, stroke, and other chronic diseases [4-7]. From a clinical perspective, it is necessary to evaluate and manage walking ability as a way to implement 
effective disease management for patients (particularly the elderly) with chronic diseases that may require HD.

Overall, survival of patients with end-stage renal dysfunction has been improving annually over the past decade, regardless of whether HD or peritoneal dialysis is provided as treatment. This is because dialysis treatment technology and disease management for patients undergoing dialysis have made great progress in recent years [8]. Despite this, the percentage of elderly HD patients who are 60 years or older has reached $75 \%$ in Japan [9]. Accordingly, HD patients are more likely to have decreased walking speed due to age-related loss of muscle mass (sarcopenia), as well as decreased muscle strength and balance function due to reduced physical activity [10]. In addition, patients who have undergone HD for 10 years or more comprise one third of all HD patients [9]. After regular HD treatment is initiated, these patients are also more likely to develop multiple comorbidities including ischemic heart disease, peripheral vascular disease, stroke, and orthopedic disease, to name a few, in addition to the primary cause of their end-stage renal dysfunction. Thus, HD patients have a 10 to 20 times higher risk for cardio-cerebrovascular events compared to age-matched healthy people [11]. However, very few studies have conducted longitudinal evaluations of walking speed associated with subsequent clinical events in HD patients, and optimal management techniques for walking speed in these patients remain unclear. Given the above, we conducted a 7-year cohort study to evaluate a range of walking speeds with regard to their capacity to reduce cardio-cerebrovascular events in HD patients.

\section{Methods}

\section{Study population}

This study was approved by the Ethics Committee of Kitasato University School of Allied Health Sciences and was conducted in accordance with the standards set forth by the latest revision of the Declaration of Helsinki. All patients received a detailed explanation of the study protocol and provided informed consent. From October 2002 to September 2014, clinically stable outpatients at the Hemodialysis Center at Sagami Junkanki Clinic were assessed for eligibility for inclusion in this prospective study. Patients were undergoing maintenance HD therapy three times a week according to the Japanese Society for Dialysis Therapy guidelines. In order to focus specifically on how walking ability impacts subsequent cardiocerebrovascular events, subjects were excluded if they fulfilled the following criteria: duration of maintenance $\mathrm{HD} \leq 3$ months; hospitalization $\leq 6$ months prior to study enrollment; history of recent myocardial infarction or angina pectoris; presence of uncontrolled cardiac arrhythmias, hemodynamic instability, or uncontrolled hypertension; require walking assistance; and the presence of any other conditions that limited walking (e.g., dementia, low vision or blindness, paralysis due to stroke, leg amputation).

\section{Demographic and clinical factors}

Information on demographic factors (age, sex, HD duration), physical characteristics (body mass index [BMI]), primary kidney disease, and comorbid conditions was collected at study entry. Blood hemoglobin, serum albu$\mathrm{min}$, and serum C-reactive protein were obtained from patient hospital charts. To quantify comorbid illnesses in this study, we used a comorbidity index developed for dialysis patients (composed of primary causes of endstage renal disease; atherosclerotic heart disease, congestive heart failure, cerebrovascular accident/transient ischemic attack, peripheral vascular disease, dysrhythmia, and other cardiac disease; chronic obstructive pulmonary disease; gastrointestinal bleeding; liver disease; cancer; and diabetes). This score was calculated using a previously described method that has been used to analyze survival among HD patients [12].

\section{Maximum walking speed}

Self-selected maximum walking speed (MWS) along a 10-m walkway was measured to assess patient walking ability before dialysis on a dialysis day. The validity and reliability of the $10-\mathrm{m}$ walk test, which measures the time (in seconds or minutes) required for a patient to walk $10 \mathrm{~m}$, have been investigated in several populations including healthy elderly, patients with neurological disorders, and those with orthopedic dysfunction [13]. First, patients were asked to walk down a $10-\mathrm{m}$ walking lane with an acceleration area at their usual speed to prepare for the MWS measurement. They were then instructed to walk as fast as was safely possible, without running. MWS was defined as the higher value of two attempts and expressed as the ratio of distance to time $(\mathrm{m} / \mathrm{min})[13]$.

\section{Cardio-cerebrovascular events}

We assessed cardio-cerebrovascular events as an indicator of subsequent adverse outcomes for HD patients. $\mathrm{Pa}$ tients were followed until the following clinical events occurred during the 7-year cohort study: hospital admission for angina, myocardial infarction, heart failure, cerebral infarction, or cerebral hemorrhage. Diagnoses for primary admission were recorded using the International Classification of Disease, version 10 (ICD-10). Angina, myocardial infarction, and heart failure were defined as ICD-10 codes I20-I22 and I50, respectively, and cerebral infarction and cerebral hemorrhage as ICD-10 codes I60-I63. Angina was defined using ICD-10 codes as follows: I20, angina pectoris; I20.0, unstable angina; I20.1, angina pectoris with documented spasm; I20.8, other 
forms of angina pectoris; and I20.9, angina pectoris, unspecified. Myocardial infarction was defined using ICD10 codes as follows: I21, acute myocardial infarction; I22, subsequent myocardial infarction; I21.0, acute transmural myocardial infarction of anterior wall; I21.1, acute transmural myocardial infarction of inferior wall; I21.2, acute transmural myocardial infarction of other sites; I21.3, acute transmural myocardial infarction of unspecified site; I21.4, acute subendocardial myocardial infarction; I21.9, acute myocardial infarction unspecified; I22.0, subsequent myocardial infarction of anterior wall; I22.1, subsequent myocardial infarction of inferior wall; I22.8, subsequent myocardial infarction of other sites; and I22.9, subsequent myocardial infarction of unspecified site. Heart failure was defined using ICD-10 codes as follows: I50, heart failure; I50.0, congestive heart failure; I50.1, congestive heart failure; and I50.9, heart failure, unspecified.

\section{Statistical analysis}

As there are no established MWS categories for HD patients, MWS was categorized into sex-specific quartiles based on previous studies that found a strong association between MWS and sex. Patients were divided into following quartiles, ranging from Q1 (slow MWS) to Q4 (fast MWS): $<79,79$ to 89,89 to 105 , and $\geq 105 \mathrm{~m} / \mathrm{min}$ in men and $<72,72$ to 85,85 to 100 , and $\geq 100 \mathrm{~m} / \mathrm{min}$ in women. Differences in clinical characteristics and MWS between quartiles were tested by one-way analysis of variance, Kruskal-Wallis test, or chi-squared test. A multivariable analysis was performed using the Cox proportional hazards regression model to estimate the independent prognostic effect of MWS on risk of subsequent cardio-cerebrovascular events after adjusting for confounders. We included the confounders (MWS, age, sex, BMI, HD duration, diabetic nephropathy of primary kidney disease, comorbidity score, hemoglobin, serum albumin, and serum C-reactive protein) in the models. Within the present study sample, there were 42 cardiocerebrovascular events, which allowed for a maximum of four variables to be included in the multivariate Cox model. If the multivariate Cox model includes more than four covariates, it has the potential to cause overfitting. To avoid overfitting, all potential confounding factors were reduced to four composite characteristics with a significant $(P<0.05)$ association with subsequent cardio-cerebrovascular events in the univariate Cox model. We calculated the cumulative incidence of cardiocerebrovascular events according to MWS quartiles. Kaplan-Meier analysis was used to estimate long-term effects. The log-rank test was used to examine differences in the risk of subsequent cardio-cerebrovascular events between MWS quartiles. The 7-year cumulative incidence probability was estimated using the life table method with the interval length set at 1 month. All analyses were performed using the Statistical Package for Social Sciences (SPSS Statistics 22.0 for Mac; IBM Corp., Armonk, NY, USA). Statistical significance was set at $P<0.05$.

\section{Results}

\section{Patient characteristics}

Of the 465 Japanese outpatients assessed for their eligibility for inclusion, we excluded 119 patients who did not satisfy the inclusion criteria, and 158 patients declined to participate in the study. Ultimately, 188 HD patients were recruited (Fig. 1). Demographic and clinical characteristics of the patients are summarized in Table 1. The patients comprised 90 men and 98 women, aged 35-88 years (mean age, 65 years). The HD duration was 3.3 (25th, 75th percentiles, 1.4, 9.5) years at baseline. The most common underlying kidney diseases were diabetic nephropathy (33.5\%), followed by glomerulonephritis (32.4\%). Mean comorbidity score was $4.8 \pm 3.0$, and MWS for all patients was $87.5 \pm 19.6 \mathrm{~m} / \mathrm{min}$. Table 1 shows the baseline characteristics of the patients according to MWS quartiles 1-4. We found significant quartiledependent differences in age, comorbidity score, serum albumin, and MWS $(P<0.01, \quad P<0.01$, $P=0.02, P<0.001$ respectively). Other baseline characteristics did not differ significantly across the four quartiles.

\section{Outcomes}

Patients were followed for up to 7 years. The overall follow-up durations ranged from 1 to 84 months (median, 53 months). During the follow-up period, cardiocerebrovascular events occurred in 42 patients (22\%): 8 of angina, 12 of myocardial infarction, 16 of heart failure, 1 of subarachnoid hemorrhage, 1 of intracerebral hemorrhage, and 4 of cerebral infarction. Twenty-five percent of the patients with Q1 and Q2 group occurred events after 21 and 40 months. On the other hand, the events rate of patients with Q3 and Q4 at the end of the follow-up was less than $25 \%$.

\section{The relationship between outcomes and MWS as continuous variables}

According to the Cox proportional hazards model, the increase in crude hazard ratio (HR) of MWS per $10 \mathrm{~m} /$ min was 0.74 (95 \% confidence interval [CI], 0.63-0.86; $P<0.001)$, which indicated that maintaining a higher MWS was associated with a reduction in cardio-cerebrovascular events (Table 2). After adjusting for the effects of age, diabetic nephropathy, and comorbidity score, the HR was 0.77 (95 \% CI, 0.65-0.92; $P=0.004$ ) (Table 2). 


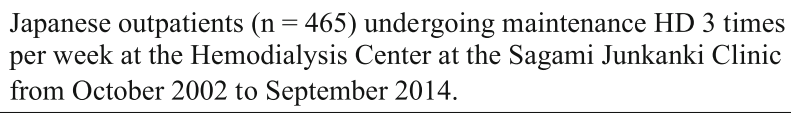

Fig. 1 Flow diagram for patient selection and exclusion

\section{The relationship between outcomes and MWS as categorical variables}

Seven-year cumulative incidence rates were 36, 32, 13, and $9 \%$ for Q1 through Q4, respectively $(P<0.001)$. The cumulative incidence rates of cardio-cerebrovascular events in Q1 were significantly higher than those in Q3 and Q4 $(P=0.003, P<0.001$, respectively). Cumulative incidence rates of cardio-cerebrovascular events in Q2 were also significantly higher than rates in Q3 and Q4 $(P=0.03, P=0.004$, respectively). While incidence rates did not significantly differ between Q1 and Q2 or between Q3 and Q4, Kaplan-Meier curves showed clear differences between Q2 and Q3 (Fig. 2).

\section{Discussion}

The main finding of this study is that the initial MWS at the time of study entry significantly influenced the 7year cumulative incidence of cardio-cerebrovascular events in ambulatory patients on hemodialysis (HD), independent of age, sex, BMI, HD duration, diabetic nephropathy of primary kidney disease, comorbid conditions, and markers of anemia, nutrition, and inflammation. To our knowledge, this is the first study to demonstrate an association between walking ability and subsequent cardio-cerebrovascular events in an Asian population of patients with chronic kidney disease undergoing HD. Based on our findings, the hazard ratio for clinical events per $10-\mathrm{m} / \mathrm{min}$ increases in MWS was 0.77 . Our study also revealed that male HD patients with MWS $\geq 89 \mathrm{~m} / \mathrm{min}$ and female HD patients with MWS $\geq 85 \mathrm{~m} / \mathrm{min}$ at baseline had a significantly lower cumulative incidence of cardio-cerebrovascular events compared to other patients during the 7-year follow-up period. These different walking speeds had not yet been evaluated for their potential to reduce subsequent clinical events in ambulatory HD patients. As such, our present study findings have important implications with regard to effective disease management in Japanese HD populations.

Recent studies have shown that walking speed is gaining interest for the prognosis of adverse health-related events, particularly for elderly individuals. MonteroOdasso et al. showed that a slow walking speed alone in otherwise well-functioning elderly individuals was enough to predict the risk for future adverse events during a 2-year follow-up study [5]. A recent 7-year cohort 
Table 1 Baseline characteristics based on levels of maximum walking speed

\begin{tabular}{|c|c|c|c|c|c|c|}
\hline \multirow[t]{2}{*}{ Characteristic } & \multirow{2}{*}{$\begin{array}{l}\text { Overall } \\
(n=188)\end{array}$} & \multicolumn{4}{|c|}{ Maximum walking speed } & \multirow{2}{*}{$\begin{array}{l}P \\
\text { value }\end{array}$} \\
\hline & & $\mathrm{Q} 1(n=47)$ & $\mathrm{Q} 2(n=47)$ & Q3 $(n=47)$ & $\mathrm{Q} 4(n=47)$ & \\
\hline Age (years) & $65(10)$ & $73(10)$ & $64(8)$ & $63(9)$ & $60(9)$ & $<0.001$ \\
\hline Women & $98(52.1)$ & $24(51)$ & $25(53)$ & $24(51)$ & $25(53)$ & 0.99 \\
\hline Body mass index $\left(\mathrm{kg} / \mathrm{m}^{2}\right)$ & $21.2(3.2)$ & $20.3(3.2)$ & $21.7(3.3)$ & $20.7(3.3)$ & $21.9(2.8)$ & 0.42 \\
\hline Hemodialysis duration (years) & $3.3(1.4,9.5)$ & $2.5(1.2,8.1)$ & $3.8(2.0,12.6)$ & $3.2(1.8,7.3)$ & $3.3(1.0,8.5)$ & 0.35 \\
\hline Primary kidney disease & & & & & & 0.19 \\
\hline Diabetic nephropathy & $63(33.5)$ & $17(36.2)$ & $17(36.2)$ & $16(34.0)$ & $13(27.7)$ & \\
\hline Glomerulonephritis & $61(32.4)$ & $9(19.1)$ & 15 (31.9) & $18(38.3)$ & $19(40.4)$ & \\
\hline Hypertension & $16(8.5)$ & $4(8.5)$ & $5(10.6)$ & $4(8.5)$ & $3(6.4)$ & \\
\hline Polycystic renal disease & $7(3.7)$ & $1(2.1)$ & $0(0)$ & $4(8.5)$ & $2(4.3)$ & \\
\hline Other nephropathies & $41(21.8)$ & $16(34.0)$ & $10(21.3)$ & $5(10.6)$ & $10(21.3)$ & \\
\hline \multicolumn{7}{|l|}{ Comorbid condition } \\
\hline Presence of cardiac disease ${ }^{a}$ & $91(48.4)$ & $31(66.0)$ & $20(42.6)$ & $26(55.3)$ & $14(29.8)$ & 0.003 \\
\hline Peripheral vascular disease & $20(10.6)$ & $7(3.7)$ & $5(2.7)$ & $4(2.1)$ & $4(2.1)$ & 0.72 \\
\hline Presence of diabetes mellitus & $76(40.4)$ & $19(40.4)$ & $22(46.8)$ & $18(38.3)$ & $17(36.2)$ & 0.74 \\
\hline Comorbidity score & $4.8(3.0)$ & $6.3(2.8)$ & $4.7(3.0)$ & $4.4(2.9)$ & $3.6(2.7)$ & $<0.001$ \\
\hline \multicolumn{7}{|l|}{ Laboratory values } \\
\hline Hemoglobin (g/dL) & $10.3(1.0)$ & $10.2(0.9)$ & $10.0(1.0)$ & $10.3(0.9)$ & $10.4(1.0)$ & 0.34 \\
\hline Serum albumin (g/dL) & $3.9(0.3)$ & $3.8(0.3)$ & $3.8(0.3)$ & $3.9(0.3)$ & $4.0(0.3)$ & 0.02 \\
\hline Serum C-reactive protein (mg/dL) & $0.3(0.7)$ & $0.4(0.8)$ & $0.3(0.7)$ & $0.3(0.7)$ & $0.3(0.4)$ & 0.61 \\
\hline \multicolumn{7}{|l|}{ Walking ability } \\
\hline Maximum walking speed (m/min) & 87.5 (19.6) & $62.7(11.6)$ & $81.4(4.9)$ & $94.1(5.6)$ & $111.9(8.1)$ & $<0.001$ \\
\hline
\end{tabular}

Values are expressed as mean (standard deviation), median (25th, 75th percentiles), or number (percentage of total). The cutoff values defining the maximum walking speed quartiles were $<79,79-89,89-105$, and $\geq 105 \mathrm{~m} / \mathrm{min}$ in men and $<72,72-85,85-100$, and $\geq 100 \mathrm{~m} / \mathrm{min}$ in women

${ }^{a}$ History of coronary disease, congestive heart failure, myocardial infarction, or other cardiac disease

Table 2 Univariate and multivariate Cox models for the effects of maximum walking speed on cardio-cerebrovascular events

\begin{tabular}{|c|c|c|c|c|c|}
\hline \multirow[t]{2}{*}{ Factors } & \multirow{2}{*}{$\begin{array}{l}\text { Units of } \\
\text { increase }\end{array}$} & \multicolumn{2}{|c|}{ Univariate Cox model $^{a}$} & \multicolumn{2}{|c|}{ Multivariate Cox model $^{b}$} \\
\hline & & $\mathrm{HR}(95 \% \mathrm{Cl})$ & $P$ & $\mathrm{HR}(95 \% \mathrm{Cl})$ & $P$ \\
\hline Maximum walking speed & $10 \mathrm{~m} / \mathrm{min}$ & $0.74(0.63-0.86)$ & $<0.001$ & $0.77(0.65-0.92)$ & 0.004 \\
\hline Age (years) & 1 year & $1.05(1.01-1.08)$ & 0.006 & $1.03(0.99-1.07)$ & 0.16 \\
\hline Men (vs. women) & - & $0.60(0.33-1.11)$ & 0.10 & & \\
\hline Body mass index & $1 \mathrm{~kg} / \mathrm{m}^{2}$ & $1.03(0.93-1.13)$ & 0.61 & & \\
\hline Hemodialysis duration & 1 year & $1.01(0.97-1.05)$ & 0.70 & & \\
\hline Diabetic nephropathy (presence) & 1 presence & $1.96(1.06-3.60)$ & 0.03 & $1.93(1.02-3.64)$ & 0.04 \\
\hline Comorbidity score & 1 & $1.11(1.01-1.21)$ & 0.04 & $1.02(0.92-1.14)$ & 0.70 \\
\hline Hemoglobin & $0.1 \mathrm{~g} / \mathrm{dL}$ & $1.15(0.85-1.56)$ & 0.35 & & \\
\hline Serum albumin & $0.1 \mathrm{~g} / \mathrm{dL}$ & $0.42(0.17-1.05)$ & 0.06 & & \\
\hline Serum C-reactive protein & $0.1 \mathrm{mg} / \mathrm{dL}$ & $1.21(0.85-1.73)$ & 0.30 & & \\
\hline
\end{tabular}

Analyses were performed using a Cox proportional hazards regression model

$H R$ hazard ratio, $\mathrm{Cl}$ confidence interval

a Unadjusted for clinicopathological factors of cardio-cerebrovascular events

${ }^{\mathrm{b}}$ Adjusted for age, diabetic nephropathy, and comorbidity score 


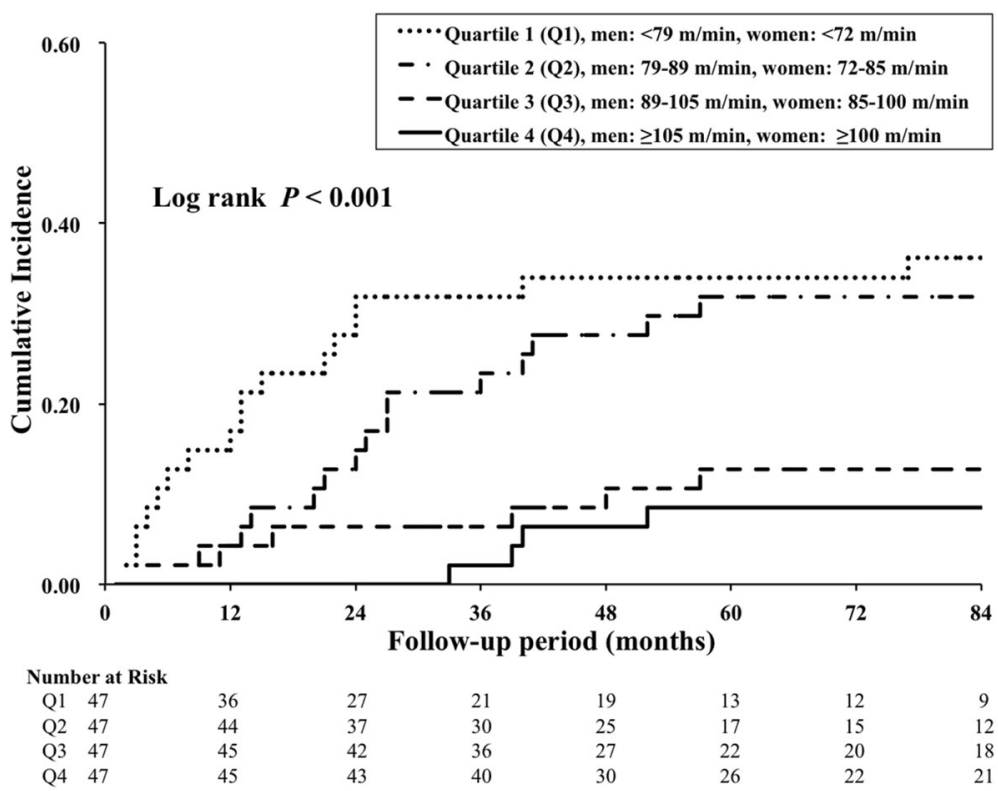

Fig. 2 Kaplan-Meier estimates for cumulative incidence rates of subsequent cardio-cerebrovascular events according to maximum walking speed quartiles

study of MWS in community-dwelling elderly reported that those who walked slowly had an almost threefold higher risk for cardiovascular mortality compared to those who walked faster [6]. Several clinical studies have found that a slow walking speed is significantly associated with an increased risk of subsequent cardiovascular events in elderly patients with ischemic heart disease, independent of cardiac function and comorbid conditions [14-17]. In addition, a slow walking speed was used as an indicator of frailty or geriatric conditions as well as decreased muscle strength (weak grip), cognitive impairment, and depression [16, 17]. Our findings are consistent with those reported by previous studies of elderly patients with chronic heart disease. Characteristics of HD patients enrolled in our present study were as follows: elderly with a mean age of 65 years (range 3588 years), long disease duration with a median time on HD of 3.3 years (range $0.3-31.2$ years), and decreased physical function with a mean MWS of $87.5 \mathrm{~m} / \mathrm{min}$ (range $33.2-139.2 \mathrm{~m} / \mathrm{min}$ ) as much as patients with chronic heart disease [14]. Physical function including walking speed among HD patients reportedly decreases year after year and tends to have already deteriorated somewhat since before the initiation of HD treatment $[18,19]$. Therefore, it is clinically necessary to evaluate and manage walking speed in order to implement effective disease management for HD patients.

The association of slow walking speed with increased risk of cardio-cerebrovascular events in the present study can be explained primarily in terms of the relationship between walking speed and physical activity.
Those who walk fast likely tend to maintain high levels of habitual physical activity of daily living. Kumahara et al. reported that walking speed was significantly associated with daily physical activity in healthy people aged 18-64 years [3]. Several other studies indicated that high levels of physical activity were associated with a decreased risk of subsequent cardio-cerebrovascular events in healthy older adults $[20,21]$. We found in previous studies that a lower level of habitual physical activity is an independent risk factor for all-cause mortality and is closely associated with slow MWS in maintenance HD patients $[13,22]$. Several previous studies also revealed that HD patients who increased their level of physical activity were able to improve risk factors for cardiocerebrovascular disease, such as hypertension, arterial stiffness, inflammatory reaction, vascular endothelial function, high levels of plasma triglycerides and low levels of high-density lipoprotein cholesterol, dysfunction of the cardiac autonomic nervous system, and reduced maximal oxygen consumption [22-24]. These reports suggest that maintaining high levels of habitual physical activity by walking at a fast speed can help prevent the development of atherosclerotic vascular disease and avoid complications from atherosclerotic vascular disease. The main findings of the present study regarding the association between slow walking speed and subsequent poor outcomes are consistent with findings noted in previous reports.

In our 7-year follow-up study, we also assessed the relationship between levels of MWS (divided into quartiles for each sex) and subsequent cardio-cerebrovascular 
events in order to clarify whether or not walking speed may comprise a part of effective disease management for the prevention of events. Kaplan-Meier curves clearly differed between Q2 and Q3 (regardless of an MWS $\geq 89 \mathrm{~m} / \mathrm{min}$ in men and $\geq 85 \mathrm{~m} / \mathrm{min}$ in women), while no significant difference was found between Q3 and Q4 (regardless of an MWS $\geq 105 \mathrm{~m} / \mathrm{min}$ in men and $\geq 100 \mathrm{~m} / \mathrm{min}$ in women). A recent cohort study of walking speed with a 5-year follow-up period reported that community-dwelling elderly people (aged $\geq 65$ years) with a slow walking speed $(\leq 90 \mathrm{~m} / \mathrm{min}$ for men and $\leq$ $81 \mathrm{~m} / \mathrm{min}$ for women) had a higher risk for cardiovascular mortality compared to those with a fast walking speed [6]. Yamamoto et al. also showed that ischemic heart disease patients with a fast walking speed $(>90 \mathrm{~m} /$ min for men and $>81 \mathrm{~m} / \mathrm{min}$ for women) at baseline had a significantly lower cumulative incidence of cardiac events compared to other participants during a 3-year follow-up study [15]. Findings from these studies are fairly consistent with our results. We would therefore surmise that MWS above that for Q3 (MWS $\geq 89 \mathrm{~m} / \mathrm{min}$ in men and $\geq 85 \mathrm{~m} / \mathrm{min}$ in women) would be a suitable walking speed for disease management in HD patients. A colleague of ours and Yamamoto have reported that the walking speed of age- and sex-matched healthy elderly people from the same geographic region as the present study was almost more than the MWS level of Q3; specifically, no healthy males had a MWS less than $90 \mathrm{~m} / \mathrm{min}$, and no healthy females had a MWS less than $81 \mathrm{~m} / \mathrm{min}$ [14]. Accordingly, anything above the Q3 MWS was thought to be a relatively attainable MWS for elderly HD patients because the lowest value within Q3 is likely to represent the lower limit for MWS among healthy elderly people. MWS above that for Q3 is also a reasonable MWS that should be achieved in order to walk to regular visits at an outpatient clinic for successful and continual HD treatment, maintain instrumental activities of daily living (e.g., outside social activities, outings, etc.), and maintain quality of life for an elderly HD outpatient. In addition, previous studies have found that leg strength and standing balance function are independently associated with walking speed in ambulatory HD patients [25]. Therefore, improvements in MWS could be achieved in HD patients showing a low MWS with exercising guidance aimed at promoting improved daily physical activity, and/or resistance training to improve leg strength and balance function.

This study had some limitations. First, because it was an observational study, residual confounders may remain. Second, given the study design, causality cannot be established. Thus, additional randomized and controlled studies are needed. To the best of our knowledge, this is the first study to show the correlation between cardio-cerebrovascular events and MWS in HD patients.
Third, because we evaluated MWS of HD patients only at baseline, temporal fluctuations in walking abilities were not evaluated. However, because we recruited clinically stable and adequately dialyzed patients, their walking ability was assumed not to fluctuate dramatically. Fourth, we excluded patients who needed assistance with walking. As a result, comorbid conditions in the patients were relatively mild. These limitations should be considered when generalizing our study results to more severely limited patients. Finally, this was a small-scale observational study, so our results may lack general applicability.

\section{Conclusions}

Our 7-year follow-up study revealed that a slow walking speed was significantly and independently associated with an increased risk of subsequent cardio-cerebrovascular events. In addition, we found that MWS $\geq 89 \mathrm{~m} / \mathrm{min}$ for males and MWS $\geq 85 \mathrm{~m} / \mathrm{min}$ for females might serve as important MWS values for disease management in HD patients.

\section{Abbreviations \\ HD: Hemodialysis; MWS: Maximum walking speed; BMI: Body mass index; ICD: International Classification of Disease; HR: Hazard ratio; Cl: confidence interval}

\section{Acknowledgements}

The authors wish to thank all patients who participated in this study. The authors also thank Dr. Yutaka Takagi, Dr. Junko Horiguchi, Ms. Junko

Yoneyama, Ms. Kaoru Uemura, Ms. Fusayo Hemmi, Mr. Naoki Murouchi, and Mr. Toshiya Minowa for performing experiments and the HD treatment staff at the Sagami Junkanki Clinic for their contributions to this study.

\section{Funding}

This research was supported by JSPS KAKENHI Grant Numbers 23500614 and 26350631.

\section{Availability of data and materials}

We decided not to share the data in our study because the data are thoroughly described and reflected in the accompanying tables and figures (all relevant data are within the paper).

\section{Authors' contributions}

This article has 11 authors. Our study needed a planner of the study (YA), three persons for the measurement (KY, MH, TW), two persons of study explainer ( $\mathrm{YT}, \mathrm{AY})$, two persons for data entry (RM, TK), two persons analyst (MK, KS), and the head of research (AM). All authors read and approved the final manuscript.

\section{Competing interests}

The authors declare that they have no competing interests.

Consent for publication

We consent to publish the entire text of my dissertation.

\section{Ethics approval and consent to participate}

This study was approved by the Ethics Committee of Kitasato University School of Allied Health Sciences and was conducted in accordance with the standards set forth by the latest revision of the Declaration of Helsinki. All patients received a detailed explanation of the study protocol and provided informed consent. 


\section{Author details}

'Department of Rehabilitation Sciences, Kitasato University Graduate School of Medical Sciences, 1-15-1 Kitasato, Sagamihara, Kanagawa 252-0373, Japan. 2Department of Rehabilitation, Kitasato University Hospital, Sagamihara, Kanagawa, Japan. ${ }^{3}$ Sagami Junkanki Clinic, Sagamihara, Kanagawa, Japan. ${ }^{4}$ Department of Rehabilitation, Kitasato University East Hospital, Sagamihara, Kanagawa, Japan. ${ }^{5}$ School of Allied Health Sciences, Kitasato University, Sagamihara, Kanagawa, Japan. ${ }^{6}$ School of Medicine, Kitasato University, Sagamihara, Kanagawa, Japan.

Received: 7 March 2016 Accepted: 20 August 2016 Published online: 23 October 2016

\section{References}

1. Painter P, Carlson L, Carey S, Paul SM, Myll J. Physical functioning and health-related quality-of-life changes with exercise training in hemodialysis patients. Am J Kidney Dis. 2000;35(3):482-92.

2. Johansen KL, Painter P, Kent-Braun JA, Ng AV, Carey S, Da Silva M, et al. Validation of questionnaires to estimate physical activity and functioning in end-stage renal disease. Kidney Int. 2001;59(3):1121-7.

3. Kumahara $H$, Schutz $Y$, Ayabe M, Yoshioka M, Yoshitake $Y$, Shindo M, et al. The use of uniaxial accelerometry for the assessment of physical-activityrelated energy expenditure: a validation study against whole-body indirect calorimetry. Br J Nutr. 2004;91(2):235-43.

4. Shinkai S, Watanabe S, Kumagai S, Fujiwara Y, Amano H, Yoshida H, et al. Walking speed as a good predictor for the onset of functional dependence in a Japanese rural community population. Age Ageing. 2000;29(5):441-6.

5. Montero-Odasso M, Schapira M, Soriano ER, Varela M, Kaplan R, Camera LA, et al. Gait velocity as a single predictor of adverse events in healthy seniors aged 75 years and older. J Gerontol A Biol Sci Med Sci. 2005;60(10):1304-9.

6. Dumurgier J, Elbaz A, Ducimetière $P$, Tavernier $B$, Alpèrovitch $A$, Tzourio C. Slow walking speed and cardiovascular death in well functioning older adults: prospective cohort study. BMJ. 2009;339:b4460. doi:10. 1136/bmj.b4460.

7. Creaby MW, Bennell KL, Hunt MA. Gait differs between unilateral and bilateral knee osteoarthritis. Arch Phys Med Rehabil. 2012;93(5):822-7. doi:10.1016/j.apmr.2011.11.029.

8. Nakai S, Masakane I, Shigematsu T, Hamano T, Yamagata K, Watanabe $Y$, et al. An overview of regular dialysis treatment in Japan (as of 31 December 2007). Ther Apher Dial. 2009;13(6):457-504. doi:10.1111/j. 1744-9987.2009.00789.x.

9. Hanafusa N, Nakai S, Iseki K, Tsubakihara Y. Japanese society for dialysis therapy renal data registry - a window through which we can view the details of Japanese dialysis population. Kidney Int Suppl (2011). 2015;5(1):15-22.

10. Cheema B, Abas H, Smith B, O'Sullivan AJ, Chan M, Patwardhan A, et al. Investigation of skeletal muscle quantity and quality in end-stage renal disease. Nephrology (Carlton). 2010;15(4):454-63. doi:10.1111/j.1440-1797. 2009.01261.x.

11. Foley RN, Parfrey PS, Harnett JD, Kent GM, O'Dea R, Murray DC, et al. Mode of dialysis therapy and mortality in end-stage renal disease. J Am Soc Nephrol. 1998;9(2):267-76.

12. Liu J, Huang Z, Gilbertson DT, Foley RN, Collins AJ. An improved comorbidity index for outcome analyses among dialysis patients. Kidney Int. 2010;77(2):141-51. doi:10.1038/ki.2009.413.

13. Kutsuna T, Matsunaga A, Matsumoto T, Ishii A, Yamamoto K, Hotta K, et al. Physical activity is necessary to prevent deterioration of the walking ability of patients undergoing maintenance hemodialysis. Ther Apher Dial. 2010; 14(2):193-200. doi:10.1111/j.1744-9987.2009.00750.x.

14. Yamamoto S, Matsunaga A, Kamiya K, Miida K, Ebina Y, Hotta K, et al. Walking speed in patients with first acute myocardial infarction who participated in a supervised cardiac rehabilitation program after coronary intervention. Int Heart J. 2012;53(6):347-52.

15. Yamamoto S, Matsunaga A, Wang G, Hoshi K, Kamiya K, Noda C, Kimura M, et al. Effect of balance training on walking speed and cardiac events in elderly patients with ischemic heart disease. Int Heart J. 2014;55(5):397-403.

16. Matsuzawa Y, Konishi M, Akiyama E, Suzuki H, Nakayama N, Kiyokuni M, et al. Association between gait speed as a measure of frailty and risk of cardiovascular events after myocardial infarction. J Am Coll Cardiol. 2013; 61(19):1964-72. doi:10.1016/j.jacc.2013.02.020.
17. Chaudhry SI, McAvay G, Chen S, Whitson H, Newman AB, Krumholz HM, et al. Risk factors for hospital admission among older persons with newly diagnosed heart failure: findings from the Cardiovascular Health Study. J Am Coll Cardiol. 2013;61(6):635-42. doi:10.1016/j.jacc.2012.11.027.

18. Painter P, Marcus RL. Assessing physical function and physical activity in patients with CKD. Clin J Am Soc Nephrol. 2013;8(5):861-72.

19. Roshanravan B, Robinson-Choen C, Patel KV, Ayers E, Littman AJ, de Boer $\mathbb{H}_{\text {, }}$ et al. Association between physical performance and all-cause mortality in CKD. J Am Soc Nephrol. 2013;24(5):822-30. doi:10.1681/ASN.2012070702.

20. Nocon M, Hiemann T, Müller-Riemenschneider F, Thalau F, Roll S, Willich SN. Association of physical activity with all-cause and cardiovascular mortality: a systematic review and meta-analysis. Eur J Cardiovasc Prev Rehabil. 2008; 15(3):239-46. doi:10.1097/HJR.0b013e3282f55e09.

21. Kono Y, Yamada S, Kamisaka K, Araki A, Fujioka Y, Yasui K, et al. Recurrence risk after noncardioembolic mild ischemic stroke in a Japanese population. Cerebrovasc Dis. 2011;31(4):365-72. doi:10.1159/000323233.

22. Matsuzawa R, Matsunaga A, Wang G, Kutsuna T, Ishii A, Abe $Y$, et al. Habitual physical activity measured by accelerometer and survival in maintenance hemodialysis patients. Clin J Am Soc Nephrol. 2012;7(12): 2010-6. doi:10.2215/CJN.03660412.

23. Deligiannis A, Kouidi E, Tourkantonis A. Effects of physical training on heart rate variability in patients on hemodialysis. Am J Cardiol. 1999:84(2):197-202.

24. Mustata S, Chan C, Lai V, Miller JA. Impact of an exercise program on arterial stiffness and insulin resistance in hemodialysis patients. J Am Soc Nephrol. 2004;15(10):2713-8.

25. Abe Y, Matsunaga A, Matsuzawa R, Kutsuna T, Yamamoto S, Yoneki $K$, et al. Determinants of slow walking speed in ambulatory patients undergoing maintenance hemodialysis. PLoS ONE. 2016;11(3):e0151037. doi:10.1371/ journal.pone.0151037.

\section{Submit your next manuscript to BioMed Central and we will help you at every step:}

- We accept pre-submission inquiries

- Our selector tool helps you to find the most relevant journal

- We provide round the clock customer support

- Convenient online submission

- Thorough peer review

- Inclusion in PubMed and all major indexing services

- Maximum visibility for your research

Submit your manuscript at www.biomedcentral.com/submit
) Biomed Central 